University of Warwick institutional repository: http://go.warwick.ac.uk/wrap

This paper is made available online in accordance with publisher policies.

Please scroll down to view the document itself. Please refer to the repository record for this item and our policy information available from the repository home page for further information.

To see the final version of this paper please visit the publisher's website. Access to the published version may require a subscription.

Author(s): Andres Carvajal \& H Polemarkis

Article Title: Identification of Pareto-improving policies: information as the real invisible hand

Year of publication: 2008

Link to published version:

http://www.sciencedirect.com/science/article/B6VBY-4NVTB43-

5/2/5dcc7d89d30556523e9d010eb51696df 


\title{
Identification of Pareto-improving policies: information as the real invisible hand ${ }^{1}$
}

\author{
Andrés Carvajal $^{2}$ H. M. Polemarchakis ${ }^{3}$ \\ Journal of Mathematical Economics 44, 2008
}

\footnotetext{
${ }^{1}$ We thank the Guest Editor, Paolo Siconolfi, and an anonymous referee, for comments and suggestions that improved the paper. We also thank Sergio Turner and Joao Correia da Silva for interesting conversations, and seminar participants at Yale, Brown, Royal Holloway College - University of London, and the 2006 European Workshop on General Equilibrium, for helpful comments. This work was done while Carvajal was a post-doctoral fellow at the Cowles Foundation for Research in Economics, Yale University, whose generous hospitality and financial support he gratefully acknowledges.

${ }^{2}$ Corresponding author. CRETA and Department of Economics, University of Warwick, Coventry CV4 7AL, United Kingdom; Phone: +44-(0)2476-523040; Fax: +44-(0)2476-523032; e-Mail address: A.M.Carvajal@Warwick.ac.uk

${ }^{3}$ CRETA and Department of Economics, University of Warwick, Coventry CV4 7AL, United Kingdom; Phone: +44-(0)2476-150051; Fax: +44-(0)2476-523032; eMail address: H.Polemarchakis@warwick.ac.uk
} 


\begin{abstract}
Finite sets of market data may not suffice to determine Pareto-improving policies.
\end{abstract}

Key words: general equilibrium; constrained suboptimality; identification.

JEL classification numbers: D50, D60 
When competitive markets fail to exhaust gains from trade, the question arises whether it is possible to design policies that induce a Pareto improvement. The transfer paradox, introduced by Leontieff, ${ }^{1}$ illustrates the difficulties implicit in policy design: without information about fundamentals that are unobservable, such as individual preferences, the prediction of the welfare effects of economic policy is far from obvious.

The theory of general equilibrium with incomplete markets argues that the problem of policy identification is, indeed, of interest: Geanakoplos and Polemarchakis (1986) showed that, when individuals face uninsurable risks, there exist, typically, policies of asset reallocation (which subjects the design of the policy to the same financial constraints that bind the individuals in the competitive market) that make every individual in the economy ex-ante better off. $^{2}$

Early literature on the empirical structure imposed by the competitive equilibrium hypothesis at the aggregate level was understood to imply that the difficulties associated with the identification of Pareto-improving policies were insurmountable: the Sonnenschein-Mantel-Debreu theorem ${ }^{3}$ was understood to imply that no information about individual (unobservable) preferences could be elicited from aggregate data.

Very perceptively, however, Brown and Matzkin (1990 and 1996) introduced an element that was missing in the discussion of the empirical implications of the competitive equilibrium model: the effects of perturbations to individual endowments yield testable restrictions on the graph of the equilibrium correspondence of a standard exchange economy. This idea was exploited by Chiappori et al (2004), Matzkin (2005) and Carvajal and Riascos

\footnotetext{
${ }^{1}$ Leontieff (1936). Donsimoni and Polemarchakis (1994) stated the paradox in a general setting; Turner (2004) finds bounds on the amount of trade that is required, at equilibrium, for the transfer paradox to arise.

${ }^{2}$ This result, which had been suggested by Stiglitz (1982), was later refined by Citanna et al. (1998) and it was extended to other types of policy by Citanna et al. (2001).

${ }^{3}$ Sonnenschein (1974), Mantel (1974), Debreu (1974); see also Mas-Colell (1977).
} 
(2005), to show that, in economies without uncertainty, the graph of the equilibrium correspondence can be used to identify individual preferences.

The argument for identification extends to economies with uncertainty and an incomplete asset market, as was shown by Kübler et al (2002) and Carvajal and Riascos (2006); but the extension requires the observation of relatively open subsets of the graph of the correspondence.

Here, we show that finite data sets of equilibrium information may be insufficient for the identification of Pareto improving policies, let alone of preferences. We restrict our attention to the types of policies considered by the original argument of Geanakoplos and Polemarchakis (1986), and show that finite data sets need not suffice for the identification of individual marginal utilities of income in different states of the world.

The intuition for this failure of identification of Pareto-improving asset reallocations is straightforward: Pareto-improving policies exist when market incompleteness allows the vectors of marginal utilities of revenue across states of the world of different individuals to diverge from co-linearity even at equilibrium. But, with vectors of marginal utilities of revenue that are neither identified nor collinear, Pareto-improving policies are unclear: on the basis of observed data, a profile of preferences in which the policy leaves at least one individual worse off cannot be ruled out.

\section{Not everything will do}

Consider a finite, two-period economy with uncertainty. Suppose that there are $I$ individuals, $S$ states of nature and $L$ commodities, with commodity 1 acting as numèraire, and that there are $A<S$ linearly independent numèraire assets. Denote asset payoffs in state $s$ by $r_{s} \in \mathbb{R}^{A}$.

Let $\mathcal{U}$ be the class of all strongly concave and strictly monotone $\mathbf{C}^{2}$ functions $v: \mathbb{R}_{++}^{L} \rightarrow \mathbb{R}$. 
For individual $i$, contingent on state $s$, endowments are $e_{s}^{i} \gg 0$ and preferences are $u_{s}^{i} \in \mathcal{U}$. There is no date-zero consumption, so a consumption plan is $x=\left(x_{s}\right)_{s=1}^{S}$. Ex-ante preferences are $U^{i}(x)=\sum_{s=1}^{S} u_{s}^{i}\left(x_{s}\right)$.

Let $p_{s} \in \mathcal{P}=\left\{p \in \mathbb{R}_{++}^{L}: p_{1}=1\right\}$ be commodity prices contingent on state $s$, and let $p=\left(p_{s}\right)_{s=1}^{S}$. Denote asset prices by $q$, and let $z^{i}$ be individual $i$ 's portfolio of assets.

If $\left(q, p,\left(z^{i}, x^{i}\right)_{i=1}^{I}\right)$ is a financial markets equilibrium of this economy, then, generically in the space of economies, there exists a redistribution of individual asset holdings that makes every individual in the economy exante better off (Geanakoplos and Polemarchakis, 1986). That is, for some $\left(d z^{i}\right)_{i=1}^{I}$, with $\sum_{i} d z^{i}=0$, it is true that, for all $i, d U^{i}=\sum_{i} d u_{s}^{i}>0$, where $d u_{s}^{i}$ is the (spot) general equilibrium welfare effect resulting from revenue transfers $\left(r_{s} d z^{j}\right)_{j=1}^{I}$ at spot equilibrium $\left(p_{s},\left(x_{s}^{j}\right)_{j=1}^{I}\right)$ in exchange economy $\left(u_{s}^{j}, e_{s}^{j}+r_{s} z^{j}(1,0, \ldots, 0)\right)_{j=1}^{I}$.

Suppose that, after asset markets have closed, a planner who knows the asset market and who has observed asset prices, $q$, and individual portfolios, $\left(z^{i}\right)_{i=1}^{I}$, but who does not know the fundamentals of the economy, namely state-contingent endowments and preferences, attempts to design one such asset redistribution. From an application of the transfer paradox, it follows, with minor qualifications, that this task is impossible: the appropriate policy is not identified from observation of data from the asset markets alone.

Proposition 1. Information from asset markets does not identify Pare- toimproving policies. Let $\left(d z^{i}\right)_{i=1}^{I}$ be an asset redistribution: $\left.\sum_{i} d z^{i}=0\right)$; if for each state there is at least one individual whose income and utility are perturbed (namely, $r_{s} d z^{i} \neq 0$ and $d u_{s}^{i} \neq 0$ ), then there exists an alternative ex-ante economy, $\left(\left(\tilde{u}_{s}^{i}, \tilde{e}_{s}^{i}\right)_{s=1}^{S}\right)_{i=1}^{I}$, that

1. cannot be ruled out on the basis of observed data: this economy has a financial markets equilibrium, $\left(\tilde{q}, \tilde{p},\left(\tilde{z}^{i}, \tilde{x}^{i}\right)_{i=1}^{I}\right)$, that is consistent with 
the observed data in the sense that $\tilde{q}=q$ and, for every individual, $\tilde{z}^{i}=z^{i} ;$ and

2. yields the opposite welfare effects for the given policy: letting $\tilde{U}^{i}(x)=$ $\sum_{s} \tilde{u}_{s}^{i}\left(x_{s}\right)$, the ex-ante welfare effects of the given policy are, for each individual $i, d \tilde{U}^{i}=-d U^{i}{ }^{4}$

Proof. By construction, for each individual $i$, contingent on state $s$, marginal utility of revenue is $\lambda_{s}^{i}>0$ such that $D u_{s}^{i}\left(x_{s}^{i}\right)=\lambda_{s}^{i} p_{s}$, while for some $\lambda_{0}^{i}>0$, $\sum_{s} \lambda_{s}^{i} r_{s}=\lambda_{0}^{i} q$. Also, $p_{s} x_{s}^{i}=p_{s} e_{s}^{i}+r_{s} z^{i}$ and $q z^{i}=0$.

Define $b_{s}^{i}=-\frac{1}{\lambda_{s}^{i}} d u_{s}^{i}$. For each $s$, since $\sum_{i} d z^{i}=0$, it follows that $\sum_{i} b_{s}^{i}=$ 0 and, hence, by Donsimoni and Polemarchakis (1994), there exists an exchange economy $\left(f_{s}^{i} \in \mathcal{U}, \omega_{s}^{i} \gg r_{s} z^{i}(1,0, \ldots, 0)\right)_{i=1}^{I}$ and a pair $\left(\tilde{p}_{s},\left(\tilde{x}_{s}^{i}\right)_{i=1}^{I}\right)$ such that (i) $\left(\tilde{p}_{s},\left(\tilde{x}_{s}^{i}\right)_{i=1}^{I}\right)$ is (spot) equilibrium for economy $\left(f_{s}^{i}, \omega_{s}^{i}\right)_{i=1}^{I}$; (ii) $D f_{s}^{i}\left(\tilde{x}_{s}^{i}\right)=\tilde{p}_{s}$; and (iii) as a consequence of revenue transfer $\left(d \omega_{1, s}^{i}=r_{s} d z^{i}\right)_{i=1}^{I}$, spot welfare effects are $d f_{s}^{i}=b_{s}^{i}$.

Define $\tilde{u}_{s}^{i}(x)=\lambda_{s}^{i} f_{s}^{i}(x)$ and $\tilde{e}_{s}^{i}=\omega_{s}^{i}-r_{s} z^{i}(1,0, \ldots, 0)$.

By construction, $\tilde{p}_{s} \tilde{e}_{s}^{i}+r_{s} z^{i}=\tilde{p}_{s} \omega_{s}^{i}$ and $D \tilde{u}_{s}^{i}\left(\tilde{x}_{s}^{i}\right)=\lambda_{s}^{i} \tilde{p}_{s}$. Also, $\sum_{s} \lambda_{s}^{i} r_{s}=$ $\lambda_{0}^{i} q, \sum_{i} \tilde{x}_{s}^{i}=\sum_{i} \tilde{e}_{s}^{i}$ and $\sum_{i} z^{i}=0$, so $\left(q, \tilde{p},\left(z^{i}, \tilde{x}^{i}\right)_{i=1}^{I}\right)$ is a financial markets equilibrium for the alternative economy.

On the other hand, for each $s$,

$$
\sum_{s} d \tilde{u}_{s}^{i}=\sum_{s} \lambda_{s}^{i} d f_{s}^{i}=\sum_{s} \lambda_{s}^{i} b_{s}^{i}=-\sum_{s} \lambda_{s}^{i} \frac{d u_{s}^{i}}{\lambda_{s}^{i}}=-\sum_{s} d u_{s}^{i} .
$$

Hence, for a given asset reallocation, all the information available from the markets that have actually been open fails to distinguish the true econ-

\footnotetext{
${ }^{4}$ For $d \tilde{U}^{i}$, let $d \tilde{u}_{s}^{i}$ be the (spot) general equilibrium welfare effect, for individual $i$, resulting from revenue transfers $\left(r_{s} d z^{j}\right)_{j=1}^{I}$ at spot equilibrium $\left(\tilde{p}_{s},\left(\tilde{x}_{s}^{j}\right)_{j=1}^{I}\right)$ in exchange economy $\left(\tilde{u}_{s}^{j}, \tilde{e}_{s}^{j}+r_{s} z^{j}(1,0, \ldots, 0)\right)_{j=1}^{I}$, and let $d \tilde{U}^{i}=\sum_{s} d \tilde{u}_{s}^{i}$.
} 
omy from an alternative one in which the same reallocation has the opposite welfare effects. In particular, the information does not distinguish an economy in which the policy is Pareto-improving from one in which it is Pareto impairing. ${ }^{5}$

This basic result is subject to criticism if it is plausible to assume that a planner may have available information from commodity markets for: (i) multiple equilibria of the economy, or (ii) a longer history of observed data.

\section{Multiple observations}

Suppose now that, after asset markets have closed, when the asset redistribution is to be designed, the planner knows not only the prices and quantities of the markets that have been open, but also future state-contingent commodity prices, individual endowments and individual consumption plans. Suppose, furthermore, that multiple observations are available. When this data set is sufficiently rich so as to allow identification of individual income effects, Kübler et al (2002) and Carvajal and Riascos (2006) have shown that, under regularity conditions, unobserved fundamentals, namely preferences, can be recovered. In order to identify income effects, however, these arguments require the observation of relatively open subsets of the equilibrium manifold, which rules out the possibility of finite data sets. The question we address here is whether finite data allow for identification of policies, even if not of preferences.

Suppose that there are $T$ observations: a data set is a sequence of prices from all markets, individual plans for all variables, and individual endow-

\footnotetext{
${ }^{5}$ It should be noticed that this analysis assumes that individual endowments are unknown. This is necessary in the argument only for the purpose of satisfying nonnegativity constraints, and neither the fact that observed prices are part of a financial equilibrium for the constructed economy, nor the fact that welfare effects go in opposite directions for both economies depend on the exact value of the constructed endowments.
} 
ments in all states, $\left(q^{t}, p^{t},\left(z^{i, t}, x^{i, t}, e^{i, t}\right)_{i=1}^{I}\right)_{t=1}^{T} \cdot{ }^{6}$ Assume that $x_{s}^{i, t} \gg 0$ and $x_{s}^{i, t^{\prime}} \neq x_{s}^{i, t}$, when $t^{\prime} \neq t$, for every $i$, every $t$ and every $s$.

Suppose that after asset markets have closed for the economy with endowments $\left(e^{i, T}\right)_{i=1}^{I}$, and the observed equilibrium is $\left(q^{T}, p^{T},\left(z^{i, T}, x^{i, T}\right)_{i=1}^{I}\right)$, a Pareto-improving asset redistribution has to be designed. Suppose that it is known that every observation in the data set reflects financial markets equilibrium: for all $t$, it is known that $\left(q^{t}, p^{t},\left(z^{i, t}, x^{i, t}\right)_{i=1}^{I}\right)$ is an equilibrium for the economy with endowments $\left(e^{i, t}\right)_{i=1}^{I}$. Suppose also that, for every $s$, $\left(p_{s}^{T},\left(x_{s}^{i, T}\right)_{i=1}^{I}\right)$ is a regular equilibrium of (spot) economy $\left(u_{s}^{i}, e_{s}^{i, T}+r_{s} z^{i, T}\right)_{i=1}^{I}$.

We next show that if individual preferences are unknown, with some qualification, even all this information fails to identify Pareto-improving policies: one can find a second profile of preferences, also consistent with the data, with the property that any policy that is Pareto-improving in the first profile makes at least one consumer worse-off if real preferences are the second profile.

Denote by $\lambda_{s}^{i, t}>0$ the marginal utility of revenue for individual $i$ in state $s$, for the $t$-th observation on the data set. By definition, $D u_{s}^{i}\left(x_{s}^{i, t}\right)=\lambda_{s}^{i, t} p_{s}^{t}$. Also, let $\lambda_{0}^{i, t}>0$ such that $\sum_{s} \lambda_{s}^{i, t} r_{s}=\lambda_{0}^{i, t} q^{t}$.

Proposition 2. Suppose that for every individual, $i$, there exists a solution

$$
\left(\left(k^{i, t}>0\right)_{t=1}^{T},\left(\left(\mu_{s}^{i, t}\right)_{s=1}^{S}\right)_{t=1}^{T-1}\right)
$$

to the system of inequalities

$$
\begin{array}{ll}
k^{i, t} D u_{s}^{i}\left(x_{s}^{i, t}\right) \cdot\left(x_{s}^{i, t}-x_{s}^{i, T}\right)<\mu_{s}^{i, t}, & t \leq T-1 ; \\
\mu_{s}^{i, t}<k^{i, T} \frac{\sum_{j \neq i} \lambda_{s}^{j, T}}{\lambda_{s}^{i, T}} D u_{s}^{i}\left(x_{s}^{i, T}\right) \cdot\left(x_{s}^{i, t}-x_{s}^{i, T}\right), & t \leq T-1 ; \\
\mu_{s}^{i, t^{\prime}}<\mu_{s}^{i, t}+k^{i, t} D u_{s}^{i}\left(x_{s}^{i, t}\right) \cdot\left(x_{s}^{i, t^{\prime}}-x_{s}^{i, t}\right), & t, t^{\prime} \leq T-1, \text { with } t \neq t^{\prime} .
\end{array}
$$

${ }^{6}$ Here, $p^{t}=\left(p_{s}^{t}\right)_{s=1}^{S}, x^{i, t}=\left(x_{s}^{i, t}\right)_{s=1}^{S}$, and $e^{i, t}=\left(x_{s}^{i, t}\right)_{s=1}^{S}$. 
Then, Pareto-improving policies are not identified by the data: there exist, for every individual and state, state-contingent preferences, $\tilde{u}_{s}^{i} \in \mathcal{U}$, that

1. cannot be ruled out on the basis of observed data: for each observation, $\left(\left(q^{t}, p^{t},\left(z^{i, t}, x^{i, t}\right)_{i=1}^{I}\right)\right.$ is a financial markets equilibrium for economy $\left.\left(\left(\tilde{u}_{s}^{i}, e_{s}^{i, t}\right)_{s=1}^{S}\right)_{i=1}^{I}\right)$; and

2. yield opposite aggregate ex-ante welfare effects for every asset redistribution at observation $T$ : for every $\left(d z^{i}\right)_{i=1}^{I}$ such that $\sum_{i} d z^{i}=0$, it is true that $\sum_{i} d \tilde{U}^{i, T}=-\sum_{i} d U^{i, T}{ }^{7}$ Moreover, if there are only two individuals in the economy, ex-ante welfare effects are $\left(d \tilde{U}^{1, T}, d \tilde{U}^{2, T}\right)=$ $-\left(d U^{2, T}, d U^{1, T}\right)$.

Proof. From a solution to the corresponding system of inequalities for each individual, it follows from lemma 1 (see Appendix 1) that there exist statecontingent preferences $\tilde{u}_{s}^{i} \in \mathcal{U}$ such that:

1. For every observation $t \leq T-1$, the gradient at $x_{s}^{i, t}$ satisfies

$$
D \tilde{u}_{s}^{i}\left(x_{s}^{i, t}\right)=\frac{k^{i, t}}{k^{i, T}} D u_{s}^{i}\left(x_{s}^{i, t}\right)
$$

2. In an open neighborhood of $x_{s}^{i, T}$, the function satisfies

$$
\tilde{u}_{s}^{i}(x)=\frac{\sum_{j \neq i} \lambda_{s}^{j, T}}{\lambda_{s}^{i, T}} u_{s}^{i}(x) .
$$

Fix $t \leq T-1$. Let $\tilde{\lambda}_{s}^{i, t}=\frac{k^{i, t}}{k^{i, T}} \lambda_{s}^{i, t}$, for all $i$ and all $s=0, \ldots, S$. Given

\footnotetext{
${ }^{7}$ Here, $d U^{i, T}=\sum_{s} d u_{s}^{i, T}$ and $d \tilde{U}^{i, T}=\sum_{s} d \tilde{u}_{s}^{i, T}$, where $d u_{s}^{i, T}$ and $d \tilde{u}_{s}^{i, T}$ are the welfare effects for individual $i$, resulting from revenue transfers $\left(r_{s} d z^{j}\right)_{j=1}^{I}$ at spot equilibrium $\left(p_{s}^{T},\left(x_{s}^{j, T}\right)_{j=1}^{I}\right)$ in exchange economies $\left(u_{s}^{j}, e_{s}^{j, T}+r_{s} z^{j, T}\right)_{j=1}^{I}$ and $\left(\tilde{u}_{s}^{j}, e_{s}^{j, T}+r_{s} z^{j, T}\right)_{j=1}^{I}$, respectively.
} 
condition 1 above, we have that

$$
D \tilde{u}_{s}^{i}\left(x_{s}^{i, t}\right)=\frac{k^{i, t}}{k^{i, T}} D u_{s}^{i}\left(x_{s}^{i, t}\right)=\frac{k^{i, t}}{k^{i, T}} \lambda_{s}^{i, t} p_{s}^{t}=\tilde{\lambda}_{s}^{i, t} p_{s}^{t},
$$

while

$$
\sum_{s} \tilde{\lambda}_{s}^{i, t} r_{s}=\sum_{s} \frac{k^{i, t}}{k^{i, T}} \lambda_{s}^{i, t} r_{s}=\frac{k^{i, t}}{k^{i, T}} \sum_{s} \lambda_{s}^{i, t} r_{s}=\frac{k^{i, t}}{k^{i, T}} \lambda_{0}^{i, t} q^{t}=\tilde{\lambda}_{0}^{i, t} q^{t}
$$

from where $\left(\left(q^{t}, p^{t},\left(z^{i, t}, x^{i, t}\right)_{i=1}^{I}\right)\right.$ is an equilibrium for $\left.\left(\left(\tilde{u}_{s}^{i}, e_{s}^{i, t}\right)_{s=1}^{S}\right)_{i=1}^{I}\right)$.

To see that $\left(\left(q^{T}, p^{T},\left(z^{i, T}, x^{i, T}\right)_{i=1}^{I}\right)\right.$ is an equilibrium for $\left.\left(\left(\tilde{u}_{s}^{i}, e_{s}^{i, T}\right)_{s=1}^{S}\right)_{i=1}^{I}\right)$, let $\tilde{\lambda}_{s}^{i, T}=\sum_{j \neq i} \lambda_{s}^{j, T}$, for all $i$ and $s=0, \ldots, S$; observe that, by condition 2 ,

$$
D \tilde{u}_{s}^{i}\left(x_{s}^{i, T}\right)=\frac{\sum_{j \neq i} \lambda_{s}^{j, T}}{\lambda_{s}^{i, T}} D u_{s}^{i}\left(x_{s}^{i, T}\right)=\sum_{j \neq i} \lambda_{s}^{j, T} p_{s}^{T}=\tilde{\lambda}_{s}^{i, T} p_{s}^{T},
$$

while

$$
\sum_{s} \tilde{\lambda}_{s}^{i, T} r_{s}=\sum_{s} \sum_{j \neq i} \lambda_{s}^{j, T} r_{s}=\sum_{j \neq i} \sum_{s} \lambda_{s}^{j, T} r_{s}=\sum_{j \neq i} \lambda_{0}^{j, T} q^{T}=\tilde{\lambda}_{0}^{i, T} q^{T}
$$

Now, let $\Lambda_{s}^{T}=\sum_{i} \lambda_{s}^{i, T}$. Since, by condition 2 above, $d p_{s}^{T}$ is the same in exchange economies $\left(u_{s}^{i}, e_{s}^{i, T}+r_{s} z^{i, T}\right)_{i=1}^{I}$ and $\left(\tilde{u}_{s}^{i}, e_{s}^{i, T}+r_{s} z^{i, T}\right)_{i=1}^{I}$, it follows that

$$
d \tilde{u}_{s}^{i, T}=\frac{\sum_{j \neq i} \lambda_{s}^{j, T}}{\lambda_{s}^{i, T}} d u_{s}^{i, T}=\left(\frac{\Lambda_{s}^{T}}{\lambda_{s}^{i, T}}-1\right) d u_{s}^{i, T}
$$

and, hence,

$$
\sum_{i} d \tilde{u}_{s}^{i, T}=\Lambda_{s}^{T} \sum_{i} \frac{d u_{s}^{i, T}}{\lambda_{s}^{i, T}}-\sum_{i} d u_{s}^{i, T}=-\sum_{i} d u_{s}^{i, T}
$$


because $\sum_{i} d z^{i}=0$ implies $\sum_{i} \frac{d u_{s}^{i, T}}{\lambda_{s}^{i, T}}=0$. It then follows that

$$
\sum_{i} \sum_{s} d \tilde{u}_{s}^{i, T}=\sum_{s} \sum_{i} d \tilde{u}_{s}^{i, T}=-\sum_{s} \sum_{i} d u_{s}^{i, T}=-\sum_{i} \sum_{s} d u_{s}^{i, T}
$$

In the particular case of two individuals, with $j \neq i$, in a neighborhood of $x_{s}^{i, T}, \tilde{u}_{s}^{i}=\frac{\lambda_{s}^{j, T}}{\lambda_{s}^{i, T}} u_{s}^{i}$, while, by successive applications of Roy's identity,

$$
\begin{aligned}
d u_{s}^{i, T} & =\lambda_{s}^{i, T}\left(d p_{s}\left(e_{s}^{i, T}-x_{s}^{i, T}\right)+r_{s} d z^{i}(1,0, \ldots, 0)\right) \\
& =\lambda_{s}^{i, T}\left(d p_{s}\left(x_{s}^{j, T}-e_{s}^{j, T}\right)-r_{s} d z^{j}(1,0, \ldots, 0)\right) \\
& =-\frac{\lambda_{s}^{i, T}}{\lambda_{s}^{j, T}} d u_{s}^{j, T}
\end{aligned}
$$

so $\sum_{s} d \tilde{u}_{s}^{i, T}=\sum_{s} \frac{\lambda_{s}^{j, T}}{\lambda_{s}^{i, T}} d u_{s}^{i, T}=-\sum_{s} d u_{s}^{j, T}$.

The assumption of the proposition guarantees the second order conditions for the economy we construct, so it implies that the first order conditions are not only necessary but also sufficient, as we need. This is required because we need the ex-ante utility functions to be strongly quasiconcave: since we are assuming additive separability of the ex-ante preferences, it does not suffice for us to impose strong quasiconcavity of the state-contingent utility functions, and we do require strong concavity; but then, since we construct the alternative economy using local, noninfinitesimal perturbations of the norm of the gradient of the original preferences (around the equilibrium consumptions for the observation where the policy has to be designed), we cannot claim that the cardinal property is preserved automatically, and we need to guarantee it by making up for these perturbations, at all other observed equilibria, uniformly across states (notice that, for all $i, k^{i, t}$ is independent of $s$ ); a solution to the system of inequalities suffices for us to be able to preserve strong quasiconcavity of ex-ante preferences after the perturbations. 
For every individual $i$, the system introduced by the proposition can be rewritten as

$$
\begin{array}{ll}
k^{i, t} \lambda_{s}^{i, t} p_{s}^{t} \cdot\left(x_{s}^{i, t}-x_{s}^{i, T}\right)<\mu_{s}^{i, t}, & \text { all } s \text { and } t \leq T-1 ; \\
\mu_{s}^{i, t}<k^{i, T} \sum_{j \neq i} \lambda_{s}^{j, T} p_{s}^{T} \cdot\left(x_{s}^{i, t}-x_{s}^{i, T}\right), & \text { all } s \text { and } t \leq T-1 ; \\
\mu_{s}^{i, t^{\prime}}<\mu_{s}^{i, t}+k^{t} \lambda_{s}^{i, t} p_{s}^{t} \cdot\left(x_{s}^{i, t^{\prime}}-x_{s}^{i, t}\right), & \text { all } s \text { and } t, t^{\prime} \leq T-1, \text { with } t \neq t^{\prime} .
\end{array}
$$

This system is a finite set of polynomial inequalities, so the set containing all the values of the variables that solve it is, by definition, a semialgebraic set (see Mishra, 1993). By the Tarski-Seidenberg theorem (see theorem 8.6.6 in Mishra, 1993), its projection into the space of all variables but $\left(\left(k^{i, t}\right)_{t=1}^{T},\left(\left(\mu_{s}^{i, t}\right)_{s=1}^{S}\right)_{t=1}^{T-1}\right)$ is itself semi-algebraic. This means that, given a data set, there exists a finite set of polynomial inequalities on the (original) vectors of marginal utilities of income, for all individuals that suffices for the implication of the theorem. Since this application of the Tarski-Seidenberg theorem does not immediately tell us how to find the quantifier-free equivalent assumption (it only tells us that it exists, and that it defines a semialgebraic set), it is important to notice that the system introduced by the theorem is linear on the quantified variables, so the problem of determining whether it has a solution is equivalent to the first step of a linear programming algorithm, and can be solved in finitely many steps (Blow et al, 2006). Still, this does not rule out the possibility that there does never exist a solution to this system, in which case the theorem would be vacuous. Appendix 2 shows that this is not the case: two general conditions in which the system has a solution are given in the appendix; they show that all the variables in the system are used, and also that there may be cases in which conditions are easy to check. For instance, if $p_{s}^{T} \cdot\left(x_{s}^{i, t}-x_{s}^{i, T}\right) \leq 0$, for all $s$ and all $t \leq T-1$, with strict inequality for some $s$ and $t$, then the system does have a solution for individual $i$. That is, if for each individual $i$ and state $s$, bundle $x_{s}^{i, T}$ is revealed preferred, in the ex-post sense, to every other $x_{s}^{i, t}$, the system 
has a solution and the data fails to identify Pareto-improving policies; this is because, in this case, the required perturbations to the gradients are given around the highest 'observed' indifference surfaces and, therefore, pose no difficulty for strong concavity.

\section{Multiple observations and rationalizability}

Literature on the empirical content of theories distinguishes two problems: rationalizability (existence) and identification (uniqueness) of fundamentals consistent with observed data and theory. Typically, theoretical work concentrates on only one of the two problems and, in particular, literature on identification takes rationalizability for granted (e.g. Kübler et al (2002), Carvajal and Riascos (2006)). So far we have done the same: we have assumed the existence of a profile of preferences that explains observed data and have concentrated on finding a second profile which (i) is consistent with the data too, and (ii) gives welfare effects that are opposite to the ones given by the original profile, for any policy. This is a nonidentification result, by (i), which is stronger and of particular interest, because of (ii); yet it still assumes rationalizability.

One can study the rationalizability and identification problems simultaneously: consider the question of whether a data set can be rationalized by two profiles of preferences with the property that any policy that is Pareto efficient in one of them makes at least one individual worse off, if the real preferences are the alternative profile.

In order to answer this question, it suffices that we substitute the assumption that for some known preferences, $\left(\left(u_{s}^{i}\right)_{s=1}^{S}\right)_{i=1}^{I}$, it is true that every $\left(q^{t}, p^{t},\left(z^{i, t}, x^{i, t}\right)_{i=1}^{I}\right)$ is an equilibrium for the economy $\left.\left(\left(u_{s}^{i}, e_{s}^{i, t}\right)_{s=1}^{S}\right)_{i=1}^{I}\right)$, by an alternative condition on the data set that suffices to imply the existence of that first profile of preferences. 
If one assumes that the data set does not directly violate equilibrium conditions, ${ }^{8}$ then, using Theorem 2 in Matzkin and Richter (1991), it suffices that there exist $\lambda_{s}^{i, t}>0$ and $u_{s}^{i, t}$, for all individual, state and observation, such that $u_{s}^{i, t^{\prime}}<u_{s}^{i, t}+\lambda_{s}^{i, t} p_{s}^{t} \cdot\left(x_{s}^{i, t^{\prime}}-x_{s}^{i, t}\right)$, whenever $t \neq t^{\prime}$, and $\lambda_{0}^{i, t} q^{t}=\sum_{s} \lambda_{s}^{i, t} r_{s}{ }^{9}$

The importance of this assumption is that all these conditions, (including the ones directly observed on the data) are polynomial inequalities, so by adding them to the individual systems introduced by proposition 2 , we have a system that guarantees the existence of two profiles of preferences with opposite aggregate ex-ante welfare effects for any asset reallocation. By appealing again to the Tarski-Seidenberg theorem, it follows that this system can be replaced by a finite set of conditions purely on the data set. By the results obtained in appendix 2, it follows that such system is noncontradictory.

Corollary 1. There exists a finite,${ }^{10}$ noncontradictory system of polynomial inequalities that suffices for the existence of state-contingent preferences $u_{s}^{i} \in$ $\mathcal{U}$ and $\tilde{u}_{s}^{i} \in \mathcal{U}$, for all $i$ and $s$, satisfying that

1. for every $t$, observed $\left(q^{t}, p^{t},\left(z^{i, t}, x^{i, t}\right)_{i=1}^{I}\right)$ is equilibrium for economy $\left(\left(u_{s}^{i}, e_{s}^{i, t}\right)_{s=1}^{S}\right)_{i=1}^{I}$;

2. for every $t$, observed $\left(q^{t}, p^{t},\left(z^{i, t}, x^{i, t}\right)_{i=1}^{I}\right)$ is equilibrium for economy $\left(\left(\tilde{u}_{s}^{i}, e_{s}^{i, t}\right)_{s=1}^{S}\right)_{i=1}^{I}$;

3. for every asset reallocation, $\left(d z^{i}\right)_{i=1}^{I}$, it is true that $\sum_{i} \sum_{s} d \tilde{u}_{s}^{i, T}=$ $-\sum_{i} \sum_{s} d u_{s}^{i, T}$.

Suppose that a planner has available a finite data set of prices, profiles of endowments and trades. He may ask whether the data set can be explained by the Walrasian model and, if so, whether it can be used to identify a

\footnotetext{
${ }^{8}$ That is, if $p_{s}^{t}\left(x_{s}^{i, t}-e_{s}^{i, t}\right)=r_{s} z^{i, t}, q^{t} z^{i, t}=0, \sum_{i} x_{s}^{i, t}=\sum_{i} e_{s}^{i, t}$ and $\sum_{i} z^{i, t}=0$.

${ }^{9}$ That the $T$-th observation can be taken as a regular equilibrium follows from Brown and Sannon (2000).

${ }^{10} \operatorname{In}\left(\mathbb{R}^{A} \times \mathcal{P}^{S} \times\left(\left(\mathbb{R}_{++}^{L} \times \mathbb{R}_{++}^{L}\right)^{S} \times \mathbb{R}^{A}\right)^{I}\right)^{T}$.
} 
Pareto-improving policy. The corollary says that it may well happen that the answer to the second part of the question is negative, although the answer to the first part is affirmative.

\section{Long histories of data}

Suppose now that, unlike in the previous sections, the economy evolves over a horizon of $T$ periods, and that what one observes is the evolution of prices over one competitive equilibrium path. We now argue that in this setting the non-identification result extends, and that it is stronger in the sense that it requires no qualification.

Let $\Sigma$ be a finite tree of events. Denote by $\Sigma^{T}$ the set of terminal nodes. ${ }^{11}$ For every node $\sigma$, let $f(\sigma)$ be the set containing all its immediate successors. Denote by $\Sigma^{T-1}$ the set of pre-terminal nodes ${ }^{12}$ Let $\sigma_{0}$ be the initial node, and for every $\sigma \neq \sigma_{0}$, let $b(\sigma)$ denote its immediate predecessor.

State-contingent preferences and endowments for individual $i$ are $u_{\sigma}^{i} \in \mathcal{U}$ and $e_{\sigma}^{i} \in \mathbb{R}_{++}^{L}$. At every non-terminal node $\sigma$, there is a finite set, $A_{\sigma}$, of one-period numèraire assets: at $\sigma^{\prime} \in f(\sigma)$, the return of asset $a \in A_{\sigma}$ is $r_{\sigma^{\prime}}^{a}$. Denote $r_{\sigma}=\left(r_{\sigma}^{a}\right)_{a \in A_{b(\sigma)}}$.

At node $\sigma$, commodity prices are $p_{\sigma}$ and individual consumptions are $x_{\sigma}^{i}$. At non-terminal $\sigma$, commodity prices are $q_{\sigma}$ and individual portfolios are $z_{\sigma}^{i}$.

Suppose that a planner observes an equilibrium $\left(p, q,\left(x^{i}, z^{i}\right)_{i=1}^{I}\right)$, and suppose that at a given pre-terminal node $\sigma$, an asset redistribution is to be designed after asset markets have closed (so all that remains in the economy is one last period of trade in commodities). ${ }^{13}$ Does the information about all

\footnotetext{
${ }^{11}$ For simplicity, it is assumed that all histories leading to a terminal node have the same number of nodes.

${ }^{12}$ That is, $\sigma \in \Sigma^{T-1}$ if, and only if, $f(\sigma) \subseteq \Sigma^{T}$.

${ }^{13}$ As before, suppose that for each $\sigma^{\prime} \in f(\sigma),\left(p_{\sigma^{\prime}},\left(x_{\sigma^{\prime}}^{i}\right)_{i=1}^{I}\right)$ is a regular equilibrium of (spot) economy $\left(u_{\sigma^{\prime}}^{i}, e_{\sigma^{\prime}}^{i}+r_{\sigma^{\prime}} z_{\sigma}^{i}\right)_{i=1}^{I}$.
} 
prices and quantities, past, present and future, and even counterfactual (from states that did not or will not occur) identify Pareto improving interventions?

Proposition 3. Market information does not identify Pareto improving policies. That is, let $\left(p, q,\left(x^{i}, z^{i}\right)_{i=1}^{I}\right)$ be a financial markets equilibrium for economy $\left(\left(u_{\sigma}^{i}, e_{\sigma}^{i}\right)_{\sigma \in \Sigma}\right)_{i=1}^{I}$; there exist individual state-contingent preferences $\tilde{u}_{\sigma}^{i} \in \mathcal{U}$, for all $i$ and all $\sigma$, that

1. cannot be ruled out on the basis of observed data: observed $\left(p, q,\left(x^{i}\right.\right.$, $\left.\left.z^{i}\right)_{i=1}^{I}\right)$ is a financial markets equilibrium for $\left(\left(\tilde{u}_{\sigma}^{i}, e_{\sigma}^{i}\right)_{\sigma \in \Sigma}\right)_{i=1}^{I}$ too; and

2. give opposite aggregate welfare effects for any asset reallocation: for every preterminal node, $\sigma \in \Sigma^{T-1}$, and every asset reallocation $\left(d z_{\sigma}^{i}\right)_{i=1}^{I}{ }^{14}$ it is true that

$$
\sum_{i} \sum_{\sigma^{\prime} \in f(\sigma)} d \tilde{u}_{\sigma^{\prime}}^{i}=-\sum_{i} \sum_{\sigma^{\prime} \in f(\sigma)} d u_{\sigma^{\prime}}^{i}
$$

Proof. The argument is similar to the proof of proposition 2, so details are omitted. For each $i$, let $\lambda_{\sigma}^{i}$ be the marginal utility of income at equilibrium in state $\sigma$. Define the new preferences by

$$
\tilde{u}_{\sigma}^{i}(x)=\frac{\sum_{j \neq i} \lambda_{\sigma}^{j}}{\lambda_{\sigma}^{i}} u_{\sigma}^{i}(x)
$$

When there are only two consumers, the stronger implication that the equilibrium information fails to distinguish an economy in which a policy is Pareto-improving and one in which it is Pareto-impairing still applies. Importantly, this result requires no qualification, since utility perturbations

\footnotetext{
${ }^{14}$ As before, $\sum_{i} d z_{\sigma}^{i}=0$
} 
are given globally without compromising strong concavity. An immediate extension follows.

Corollary 2. Suppose that for every individual $i$, there are state-independent preferences $u^{i} \in \mathcal{U}$ such that state-contingent preferences are $u_{\sigma}^{i}=\pi_{\sigma}^{i} u^{i}$ for some $\pi_{\sigma}^{i}>0$. Let $\left(p, q,\left(x^{i}, z^{i}\right)_{i=1}^{I}\right)$ be a financial markets equilibrium for $\left(u^{i},\left(\pi_{\sigma}^{i}, e_{\sigma}^{i}\right)_{\sigma \in \Sigma}\right)_{i=1}^{I}$. There exist individual state-independent preferences $\tilde{u}^{i} \in \mathcal{U}$ and state weights $\tilde{\pi}_{\sigma}^{i}>0$ that

1. cannot be ruled out on the basis of observed data: observed $\left(p, q,\left(x^{i}\right.\right.$, $\left.\left.z^{i}\right)_{i=1}^{I}\right)$ is a financial markets equilibrium for $\left(\tilde{u}^{i},\left(\tilde{\pi}_{\sigma}^{i}, e_{\sigma}^{i}\right)_{\sigma \in \Sigma}\right)_{i=1}^{I}$; and

2. give opposite aggregate welfare effects for any asset reallocation: for every preterminal node, $\sigma \in \Sigma^{T-1}$, and every asset reallocation $\left(d z^{i}\right)_{i=1}^{I}$, it is true that ${ }^{15}$

$$
\sum_{i} \sum_{\sigma^{\prime} \in f(\sigma)} \tilde{\pi}_{\sigma^{\prime}}^{i} d \tilde{u}_{\sigma^{\prime}}^{i}=-\sum_{i} \sum_{\sigma^{\prime} \in f(\sigma)} \pi_{\sigma^{\prime}}^{i} d u_{\sigma^{\prime}}^{i}
$$

Proof. Define $\lambda_{\sigma}^{i}$ as in the previous proof, and let $\tilde{u}^{i}=u^{i}$ and

$$
\tilde{\pi}_{\sigma}^{i}=\frac{\sum_{j \neq i} \lambda_{\sigma}^{j}}{\lambda_{\sigma}^{i}} \pi_{\sigma}^{i} .
$$

\section{Concluding remarks}

If an economy is nonstationary, observation of all market information does not suffice to identify Pareto-improving policies. There exists a profile of

\footnotetext{
${ }^{15}$ Here, $d u_{\sigma-}^{i}$ and $\left.d \tilde{u}_{\sigma^{\prime}}^{i}\right)$ are, respectively, individual $i$ 's welfare effects resulting from revenue transfer $\left(r_{\sigma^{\prime}} d z^{j}\right)_{j=1}^{I}$, at spot equilibrium $\left(p_{\sigma^{\prime}},\left(x_{\sigma^{\prime}}^{j}\right)_{j=1}^{I}\right)$, in exchange economies $\left(u^{j}, e_{\sigma^{\prime}}^{j}+r_{\sigma^{\prime}} z^{j}(1,0, \ldots, 0)\right)_{j=1}^{I}$ and $\left(\tilde{u}^{j}, e_{\sigma^{\prime}}^{j}+r_{\sigma^{\prime}} z^{j}(1,0, \ldots, 0)\right)_{j=1}^{I}$.
} 
preferences which would have yielded the exact same equilibrium information, but for which any reallocation policy would have opposite effects: if it is Pareto-improving in the real economy, there is at least one individual who is made worse off in the other economy. The intuition is that one equilibrium is not enough to pin down the vector of marginal utilities of income at the terminal nodes for each individual. One can shuffle and add these vectors across individuals and still respect observed market behavior. The fact that one cannot rule out these different profiles of marginal utilities is relevant when they are not collinear, which is the condition under which Paretoimproving reallocations exist in the first place. The result is then most meaningful: the same feature of the equilibrium that explains the existence of Pareto-improving policies implies that it is impossible to identify one such policy.

When the data set is a series of equilibrium prices and endowments for a two-period economy, the problem is more restrictive and the nonidentification results are weakened. This is so, because the shuffling and addition of marginal utilities of income implies non-infinitesimal perturbations to the (norm of the gradient of) utility functions at the points where policy is to be attempted. When multiple equilibria have to be rationalized, these perturbations must be local. But then, since we have additively separable preferences, the necessity to guarantee concavity weakens the result. In this paper, we guarantee concavity via a system similar to Afriat inequalities. It follows that there do exist conditions on the data under which one can ensure the existence of multiple rationalizations yielding different welfare effects for any policy.

In both settings, when identification fails, market performance is less questionable on grounds of its inefficiency. Granted, there may be Paretoimproving policies, but market information does not suffice for their design.

Open questions remain: our setting does not consider Markovian econo- 
mies; we do not consider policies other than asset reallocation; by the qualification of the result in the case of multiple observations, our results cannot say what happens as one increases, asymptotically, the number of observations in a neighborhood of the equilibrium where policy is to be designed; finally, our results do not say whether or not a mechanism can be designed in order to elicit information about the consumers' preferences.

\section{Appendices}

\section{Appendix 1: Non-infinitesimal perturbation of a strongly concave function}

Lemma 1. Fix $u \in \mathcal{U}, \Lambda \in \mathbb{R}_{++}$and take a finite sequence $\left(x^{t}\right)_{t=1}^{T}$ in $\mathbb{R}_{+}^{L}$, such that $x^{t} \neq x^{t^{\prime}}$ for $t \neq t^{\prime}$. Let $\left(\left(k^{t}>0\right)_{t=1}^{T},\left(\mu^{t}\right)_{t=1}^{T-1}\right)$ solve the system

$$
\begin{array}{ll}
k^{t} D u\left(x^{t}\right) \cdot\left(x^{t}-x^{T}\right)<\mu^{t}, & \text { all } t \leq T-1 ; \\
\mu^{t}<k^{T} \Lambda D u\left(x^{T}\right) \cdot\left(x^{t}-x^{T}\right), & \text { all } t \leq T-1 ; \\
\mu^{t^{\prime}}<\mu^{t}+k^{t} D u\left(x^{t}\right) \cdot\left(x^{t^{\prime}}-x^{t}\right), & \text { all } t, t^{\prime} \leq T-1, \text { with } t \neq t^{\prime} .
\end{array}
$$

There exists $\tilde{u} \in \mathcal{U}$ such that:

1. for every $t \leq T-1, D \tilde{u}\left(x^{t}\right)=\frac{k^{t}}{k^{T}} D u\left(x^{t}\right)$;

2. there exists $\epsilon>0$ such that, for all $x \in B_{\epsilon}\left(x^{T}\right), \tilde{u}(x)=\Lambda u(x)$.

Proof. For every $t \leq T-1$, define $v^{t}=\Lambda u\left(x^{T}\right)+\frac{\mu^{t}}{k^{T}}$. By construction,

$$
\begin{array}{ll}
\Lambda u\left(x^{T}\right)<v^{t}+\frac{k^{t}}{k^{T}} D u\left(x^{t}\right) \cdot\left(x^{T}-x^{t}\right), & \text { all } t \leq T-1 ; \\
v^{t}<\Lambda u\left(x^{T}\right)+\Lambda D u\left(x^{T}\right) \cdot\left(x^{t}-x^{T}\right), & \text { all } t \leq T-1 ; \\
v^{t^{\prime}}<v^{t}+\frac{k^{t}}{k^{T}} D u\left(x^{t}\right) \cdot\left(x^{t^{\prime}}-x^{t}\right), & \text { all } t, t^{\prime} \leq T-1, \text { with } t \neq t^{\prime} .
\end{array}
$$

As in Matzkin and Richter (1991), define $h: \mathbb{R}^{L} \rightarrow \mathbb{R}_{+}$, as $h(x)=\sqrt{\|x\|^{2}+1}$ 
-1 . Function $h$ is $C^{2}$ and strongly convex, satisfies that $h(x)=0$ only for $x=0$, and the value of all its partial derivatives always lies in $[0,1)$.

Since $T$ is finite, there exists $\gamma>0$ such that

$$
\Lambda u\left(x^{T}\right)<v^{t}+\frac{k^{t}}{k^{T}} D u\left(x^{t}\right) \cdot\left(x^{T}-x^{t}\right)-\gamma h\left(x^{T}-x^{t}\right),
$$

and

$$
v^{t^{\prime}}<v^{t}+\frac{k^{t}}{k^{T}} D u\left(x^{t}\right) \cdot\left(x^{t^{\prime}}-x^{t}\right)-\gamma h\left(x^{t^{\prime}}-x^{t}\right),
$$

for all $t, t^{\prime} \leq T-1, t \neq t^{\prime}$. Further, restrict $\gamma$ to

$$
0<\gamma<\min _{l \leq L, t \leq T-1} \frac{k^{t}}{k^{T}} \frac{\partial u}{\partial x_{l}}\left(x^{t}\right)
$$

Now, for each $t \leq T-1$, define $\phi_{t}: \mathbb{R}^{L} \rightarrow \mathbb{R}$ by

$$
\phi_{t}(x)=u^{t}+\frac{k^{t}}{k^{T}} D u\left(x^{t}\right) \cdot\left(x-x^{t}\right)-\gamma h\left(x-x^{t}\right),
$$

while $\phi_{T}: \mathbb{R}^{L} \rightarrow \mathbb{R}$ is given by $\phi_{T}(x)=\Lambda u(x)$. Notice that every $\phi_{t}$ function is strictly concave, whereas, for each $t \leq T-1$ and every $l \in\{1, \ldots, L\}$,

$$
\frac{\partial \phi_{t}}{\partial x_{l}}(x)=\frac{k^{t}}{k^{T}} \frac{\partial u}{\partial x_{l}}\left(x^{t}\right)-\gamma \frac{\partial h}{\partial x_{l}}\left(x-x^{t}\right)>\frac{k^{t}}{k^{T}} \frac{\partial u}{\partial x_{l}}\left(x^{t}\right)-\gamma>0 .
$$

Define $\tilde{u}: \mathbb{R}_{+}^{L} \rightarrow \mathbb{R}$ by $\tilde{u}(x)=\min \left\{\phi_{1}(x), \ldots, \phi_{T}(x)\right\}$. Function $\tilde{u}$ is continuous, strongly concave, strictly monotone and differentiable almost everywhere. Differentiability everywhere can be obtained as in Chiappori and Rochet (1987). By continuity, for each $t$ there exists $\epsilon_{t}>0$ such that, for all $x \in B_{\epsilon_{t}}\left(x^{t}\right), u(x)=\phi_{t}(x)$. 


\section{Appendix 2: Solutions to the system in Proposition 2}

Some general cases in which the system introduced in proposition 2 has a solution are presented here.

Fix an individual, $i$, and define $\Lambda_{s}^{t}=\sum_{j \neq i} \frac{\lambda_{s}^{j, t}}{\lambda_{s}^{i, t}}$. For the sake of simplicity, ignore, from now on, the superindex $i$. Define

$$
M_{s}^{t}=\left\{t^{\prime} \leq T-1: D u_{s}\left(x_{s}^{t}\right) \cdot\left(x_{s}^{t^{\prime}}-x_{s}^{t}\right)<0\right\}
$$

and

$$
P_{s}^{t}=\left\{t^{\prime} \leq T-1: D u_{s}\left(x_{s}^{t}\right) \cdot\left(x_{s}^{t^{\prime}}-x_{s}^{t}\right)>0\right\}
$$

\section{Perturbation to the gradients at all $t$}

Define

$$
\begin{gathered}
\mathbb{M}^{t}=\left\{s: D u_{s}\left(x_{s}^{t}\right) \cdot\left(x_{s}^{T}-x_{s}^{t}\right)<0\right\}, \\
\mathbb{P}^{t}=\left\{s: D u_{s}\left(x_{s}^{t}\right) \cdot\left(x_{s}^{T}-x_{s}^{t}\right)>0\right\}, \\
\mathbf{M}=\left\{(s, t): t \leq T-1 \wedge D u_{s}\left(x_{s}^{T}\right)\left(x_{s}^{T}-x_{s}^{t}\right)<0\right\}, \\
\mathbf{P}=\left\{(s, t): t \leq T-1 \wedge D u_{s}\left(x_{s}^{T}\right)\left(x_{s}^{T}-x_{s}^{t}\right)>0\right\},
\end{gathered}
$$

and denote

$$
\begin{gathered}
L_{s}^{t}=\max _{t^{\prime} \in P_{s}^{t}} \frac{\Lambda_{s}^{t^{\prime}}\left(u_{s}\left(x_{s}^{t^{\prime}}\right)-u_{s}\left(x_{s}^{T}\right)\right)-\Lambda_{s}^{t}\left(u_{s}\left(x_{s}^{t}\right)-u_{s}\left(x_{s}^{T}\right)\right)}{D u_{s}\left(x_{s}^{t}\right) \cdot\left(x_{s}^{t^{\prime}}-x_{s}^{t}\right)}, \\
U_{s}^{t}=\min _{t^{\prime} \in M_{s}^{t}} \frac{\Lambda_{s}^{t^{\prime}}\left(u_{s}\left(x_{s}^{t^{\prime}}\right)-u_{s}\left(x_{s}^{T}\right)\right)-\Lambda_{s}^{t}\left(u_{s}\left(x_{s}^{t}\right)-u_{s}\left(x_{s}^{T}\right)\right)}{D u_{s}\left(x_{s}^{t}\right) \cdot\left(x_{s}^{t^{\prime}}-x_{s}^{t}\right)}, \\
\mathbb{L}^{t}=\max _{s \in \mathbb{M}^{t}} \Lambda_{s}^{t}, \mathbb{U}^{t}=\min _{s \in \mathbb{P}^{t}} \Lambda_{s}^{t}, \mathbf{U}=\min _{(s, t) \in \mathbf{M}} \frac{\Lambda_{s}^{t}}{\Lambda_{s}^{T}}, \text { and } \mathbf{L}=\max _{(s, t) \in \mathbf{P}} \frac{\Lambda_{s}^{t}}{\Lambda_{s}^{T}} .
\end{gathered}
$$

Suppose that

1. $\mathbf{L} \leq \mathbf{U}$; 
2. for all $t \leq T-1$ and all $s, M_{s}^{t} \cup P_{s}^{t}=\{1, \ldots, T-1\}$;

3. for all $t,\left[\mathbb{L}^{t}, \mathbb{U}^{t}\right] \cap \bigcap_{s}\left(L_{s}^{t}, U_{s}^{t}\right) \neq \varnothing$.

and let

1. $k^{T} \in[\mathbf{L}, \mathbf{U}]$;

2. for all $t \leq T-1$ and all $s, \mu_{s}^{t}=\Lambda_{s}^{t}\left(u_{s}\left(x_{s}^{t}\right)-u_{s}\left(x_{s}^{T}\right)\right)$;

3. for all $t, k^{t} \in\left[\mathbb{L}^{t}, \mathbb{U}^{t}\right] \cap \bigcap_{s}\left(L_{s}^{t}, U_{s}^{t}\right)$.

By strong concavity, $u_{s}\left(x_{s}^{t^{\prime}}\right)<u_{s}\left(x_{s}^{t}\right)+D u_{s}\left(x_{s}^{t}\right) \cdot\left(x_{s}^{t^{\prime}}-x_{s}^{t}\right)$, for all $s$ and all $t, t^{\prime} \leq T, t \neq t^{\prime}$.

Fix $s$ and $t \leq T-1$. Since $\Lambda_{s}^{t}>0$, we have that $\Lambda_{s}^{t} D u_{s}\left(x_{s}^{t}\right) \cdot\left(x_{s}^{t}-x_{s}^{T}\right)<\mu_{s}^{t}$. Then: if $D u_{s}\left(x_{s}^{t}\right) \cdot\left(x_{s}^{T}-x_{s}^{t}\right)<0$, since $k^{t} \geq \Lambda_{s}^{t}$, then $\mu_{s}^{t}>k^{t} D u_{s}\left(x_{s}^{t}\right) \cdot\left(x_{s}^{t}-x_{s}^{T}\right)$; if $D u_{s}\left(x_{s}^{t}\right) \cdot\left(x_{s}^{T}-x_{s}^{t}\right)>0$, since $k^{t} \leq \Lambda_{s}^{t}$, then $\mu_{s}^{t}>k^{t} D u_{s}\left(x_{s}^{t}\right) \cdot\left(x_{s}^{t}-x_{s}^{T}\right)$; if, alternatively, $D u_{s}\left(x_{s}^{t}\right) \cdot\left(x_{s}^{T}-x_{s}^{t}\right)=0$, then $\mu_{s}^{t}>0=k^{t} D u_{s}\left(x_{s}^{t}\right) \cdot\left(x_{s}^{t}-x_{s}^{T}\right)$. This yields the first equation of the system.

Again, since $\Lambda_{s}^{t}>0$, we have that $\mu_{s}^{t}<\Lambda_{s}^{t} D u_{s}\left(x_{s}^{T}\right) \cdot\left(x_{s}^{t}-x_{s}^{T}\right)$. Then: if $D u_{s}\left(x_{s}^{T}\right) \cdot\left(x_{s}^{t}-x_{s}^{T}\right)>0$, since $k^{T} \geq \frac{\Lambda_{s}^{t}}{\Lambda_{s}^{T}}$, then $\mu_{s}^{t}<k^{T} \Lambda_{s}^{T} D u_{s}\left(x_{s}^{T}\right) \cdot\left(x_{s}^{t}-x_{s}^{T}\right)$; if $D u_{s}\left(x_{s}^{T}\right) \cdot\left(x_{s}^{t}-x_{s}^{T}\right)<0$, since $k^{T} \leq \frac{\Lambda_{s}^{t}}{\Lambda_{s}^{T}}$, then $\mu_{s}^{t}<k^{T} \Lambda_{s}^{T} D u_{s}\left(x_{s}^{T}\right) \cdot\left(x_{s}^{t}-x_{s}^{T}\right)$; if, alternatively, $D u_{s}\left(x_{s}^{T}\right) \cdot\left(x_{s}^{t}-x_{s}^{T}\right)=0$, then $\mu_{s}^{t}<0=k^{T} \Lambda_{s}^{T} D u_{s}\left(x_{s}^{T}\right) \cdot\left(x_{s}^{t}-x_{s}^{T}\right)$. This yields the second equation of the system.

Now, fix $s$ and $t, t^{\prime} \leq T-1, t \neq t^{\prime}$. If $D u_{s}\left(x_{s}^{t}\right) \cdot\left(x_{s}^{t^{\prime}}-x_{s}^{t}\right)>0$, since

$$
k^{t}>\frac{\Lambda_{s}^{t^{\prime}}\left(u_{s}\left(x_{s}^{t^{\prime}}-u_{s}\left(x_{s}^{T}\right)\right)-\Lambda_{s}^{t}\left(u_{s}\left(x_{s}^{t}-u_{s}\left(x_{s}^{T}\right)\right)\right.\right.}{D u_{s}\left(x_{s}^{t}\right) \cdot\left(x_{s}^{t^{\prime}}-x_{s}^{t}\right)},
$$

it follows by construction that $\mu_{s}^{t^{\prime}}<\mu_{s}^{t}+k^{t} D u_{s}\left(x_{s}^{t}\right) \cdot\left(x_{s}^{t^{\prime}}-x_{s}^{t}\right)$. If, on the other hand, $D u_{s}\left(x_{s}^{t}\right) \cdot\left(x_{s}^{t^{\prime}}-x_{s}^{t}\right)<0$, since

$$
k^{t}<\frac{\Lambda_{s}^{t^{\prime}}\left(u_{s}\left(x_{s}^{t^{\prime}}-u_{s}\left(x_{s}^{T}\right)\right)-\Lambda_{s}^{t}\left(u_{s}\left(x_{s}^{t}-u_{s}\left(x_{s}^{T}\right)\right)\right.\right.}{D u_{s}\left(x_{s}^{t}\right) \cdot\left(x_{s}^{t^{\prime}}-x_{s}^{t}\right)},
$$


it follows again that $\mu_{s}^{t^{\prime}}<\mu_{s}^{t}+k^{t} D u_{s}\left(x_{s}^{t}\right) \cdot\left(x_{s}^{t^{\prime}}-x_{s}^{t}\right)$. Finally, notice that $D u_{s}\left(x_{s}^{t}\right) \cdot\left(x_{s}^{t^{\prime}}-x_{s}^{t}\right)=0$ does not occur, by assumption. This yields the third condition of the system.

\section{Perturbation to the gradients at $T$ only}

Suppose that there are $s$ and $s^{\prime}$ such that $P_{s}^{T} \neq \varnothing$ and $M_{s}^{T} \neq \varnothing$, and denote that

$$
L=\max _{s: P_{s}^{T} \neq \varnothing} \max _{t \in P_{s}^{T}} \frac{u_{s}\left(x_{s}^{t}\right)-u_{s}\left(x_{s}^{T}\right)}{\Lambda_{s}^{T} D u_{s}\left(x_{s}^{T}\right) \cdot\left(x_{s}^{t}-x_{s}^{T}\right)}
$$

and

$$
U=\min _{s: M_{s}^{T} \neq \varnothing} \min _{t \in M_{s}^{T}} \frac{u_{s}\left(x_{s}^{t}\right)-u_{s}\left(x_{s}^{T}\right)}{\Lambda_{s}^{T} D u_{s}\left(x_{s}^{T}\right) \cdot\left(x_{s}^{t}-x_{s}^{T}\right)} .
$$

Suppose that $L<U$. Let

1. $k^{T} \in(\max \{0, L\}, U)$;

2. for all $t \leq T-1$ and all $s$, let $\mu_{s}^{t}=u_{s}\left(x_{s}^{t}\right)-u_{s}\left(x_{s}^{T}\right)$;

3. for all $t, k^{t}=1$.

Using strong concavity, the first and third conditions of the system are immediate. Now, fix $s$ and $t \leq T-1$. If $D u_{s}\left(x_{s}^{T}\right) \cdot\left(x_{s}^{t}-x_{s}^{T}\right)>0$, then

$$
k^{T}>\frac{u_{s}\left(x_{s}^{t}\right)-u_{s}\left(x_{s}^{T}\right)}{\Lambda_{s}^{T} D u_{s}\left(x_{s}^{T}\right) \cdot\left(x_{s}^{t}-x_{s}^{T}\right)},
$$

so $\mu_{s}^{t}<k^{T} \Lambda_{s}^{T} D u_{s}\left(x_{s}^{T}\right) \cdot\left(x_{s}^{t}-x_{s}^{T}\right)$. If $D u_{s}\left(x_{s}^{T}\right) \cdot\left(x_{s}^{t}-x_{s}^{T}\right)<0$, then

$$
k^{T}<\frac{u_{s}\left(x_{s}^{t}\right)-u_{s}\left(x_{s}^{T}\right)}{\Lambda_{s}^{T} D u_{s}\left(x_{s}^{T}\right) \cdot\left(x_{s}^{t}-x_{s}^{T}\right)}
$$

so $\mu_{s}^{t}<k^{T} \Lambda_{s}^{T} D u_{s}\left(x_{s}^{T}\right) \cdot\left(x_{s}^{t}-x_{s}^{T}\right)$. Finally, if $D u_{s}\left(x_{s}^{T}\right) \cdot\left(x_{s}^{t}-x_{s}^{T}\right)=0$, then $u_{s}\left(x_{s}^{t}\right)<u_{s}\left(x_{s}^{T}\right)$, so $\mu_{s}^{t}=u_{s}\left(x_{s}^{t}\right)-u_{s}\left(x_{s}^{T}\right)<0=k^{T} \Lambda_{s}^{T} D u_{s}\left(x_{s}^{T}\right) \cdot\left(x_{s}^{t}-x_{s}^{T}\right)$.

This yields the second condition of the system. 
Notice that, by construction, for this case to apply it suffices that $u_{s}\left(x_{s}^{t}\right) \leq$ $u_{s}\left(x_{s}^{T}\right)$ whenever $D u_{s}\left(x_{s}^{T}\right) \cdot\left(x_{s}^{t}-x_{s}^{T}\right)>0$. Notice also that the solution can easily be given if $P_{s}^{T}=\varnothing$, for all $s$, but $M_{s^{\prime}}^{T} \neq \varnothing$ for some $s^{\prime}$, or if $M_{s}^{T}=\varnothing$, for all $s$, but $P_{s^{\prime}}^{T} \neq \varnothing$ for some $s^{\prime}$.

\section{References}

Blow L, Browning M, Crawford I. Revealed preference analysis of characteristics models. Mimeo 2006.

Brown D, Matzkin R. Recoverability and estimation of the demand and utility functions of traders when demands are unobservable. Mimeo 1990.

Brown D, Matzkin R. Testable restrictions on the equilibrium manifold. Econometrica 1996; 64; 1249-1262.

Brown D, Shannon C. Uniqueness, stability and comparative statics in rationalizable Walrasian Markets. Econometrica 2000; 68; 1529-1539.

Carvajal A, Riascos A. Identification of preferences from market data. Advances in Theoretical Economics 2005; 5; 3.

Carvajal A, Riascos A. Identification of preferences from market data under uncertainty. Mimeo 2006.

Chiappori P-A, Ekeland I, Kubler F, Polemarchakis H. Testable implications of general equilibrium theory: a differentiable approach. Journal of Mathematical Economics 2004; 40; 105-120.

Chiappori P-A, Rochet J-C. Revealed preferences and differentiable demand. Econometrica 1987; 55; 687-692.

Citanna A, Kajii A, Villanacci A. Constrained suboptimality in incomplete markets: a general approach and two applications. Economic Theory 1998; 11; 495-521.

Citanna A, Polemarchakis H, Tirelli M. The taxation of trades in assets. Journal of Economic Theory 2006; 126; 299-313. 
Debreu G. Excess demand functions. Journal of Mathematical Economics $1974 ; 1 ; 15-23$.

Donsimoni MP, Polemarchakis H. Redistribution and welfare. Journal of Mathematical Economics 1994; 23; 235-242.

Geanakoplos J, Polemarchakis H. Existence, regularity and constrained suboptimality of competitive allocations when assets structure is incomplete. In Heller W, Starr R, Starret D (Eds), Essays in honor of Kenneth J. Arrow, Vol 3. Cambridge; 1986; 65-95.

Kübler F, Chiappori P-A, Ekeland I, Polemarchakis H. The identification of Preferences from Equilibrium Prices under Uncertainty. Journal of Economic Theory 2002; 102; 403-420.

Leontieff W. Note on the pure theory of capital transfers. In Explorations in economics: notes and essays in honor of F.W. Taussig. McGraw-Hill; 1936.

Mantel, R. On the characterization of aggregate excess demand. Journal of Economic Theory 1974; 7; 348-353.

Mas-Colell, A. On the equilibrium price set of an exchange economy. Journal of Mathematical Economics 1977; 4; 117-126.

Matzkin R. Identification of consumers' preferences when their choices are unobservable. Economic Theory 2005; 25; 423-443.

Matzkin R, Richter M. Testing strictly concave rationality. Journal of Economic Theory 1991; 58; 287-303.

Mishra B. Algorithmic Algebra. Springer-Verlag: New York; 1993.

Sonnenschein, H. Market excess demand functions. Econometrica 1974; 40; 549-563.

Stiglitz J. The inefficiency of stock market equilibrium. Review of Economic Studies 1982; 49; 241-261.

Turner, S. How much trade does the transfer paradox require? The threshold computed. Mimeo 2004. 